\title{
Cognición, actitud y conducta: cambio en uDIs hacia la reducción de los riesgos asociados al uso de drogas*
}

\author{
InsúA, P.* ; MONCADA, S.* * \\ * Facultad de Psicología. Universidad del País Vasco. \\ * Plan Nacional sobre Drogas. \\ Enviar correspondencia: \\ PATRICIA INSÚA. Facultad de Psicología. Universidad del País Vasco. \\ Avda. TOLOSA, 70. 20018 San Sebastián. Teléfono: 943015735. E-mail: pbpincep@ss.ehu.es
}

\section{RESUMEN}

Objetivo: El objetivo es evaluar 15 Talleres diseñados para la reducción de los riesgos asociados a la inyección de drogas.

Material y Método: La muestra incluye 229 hombres y mujeres con una edad promedio de 34 años, policonsumidores de drogas por la vía parenteral. Los sujetos fueron evaluados en pretest, postest y seguimiento utilizando un cuestionario autoaplicado sobre conductas de riesgo asociadas a la inyección y variables mediadoras en estas conductas.

Resultados: Los Talleres de Consumo de Menor Riesgo están asociados a una disminución en las conductas de riesgo y a un aumento en las conductas de protección en la inyección de drogas. También están asociados a un aumento en los conocimientos sobre los riesgos asociados a la inyección de drogas y sobre la transmisión del VIH / SIDA, a una mayor autoeficacia de afrontamiento ante situaciones de riesgo y a un mantenimiento de la motivación para reducir estos riesgos. Adicionalmente, la satisfacción de los usuarios de estos Talleres es muy elevada.

Conclusión: Estos resultados proporcionan apoyo a la eficacia de estos Talleres de Consumo de Menor Riesgo, e indirectamente en favor del Programa de Formación en redes para profesionales sanitarios para el diseño, implementación y evaluación de los Talleres para la reducción de riesgos asociados al uso de drogas.

Palabras clave: Reducción de riesgos, UDIs, Programa, Taller, SIDA

\section{SUMMARY}

Objective: Objective is to evaluate 15 workshops designed to improve injection practices among injecting drug users.

Method: Sample includes 229 male and female injecting drug users assigned non-randomly to one comparison group or to one workshop user-group. They were assessed three times using a questionnaire including questions on injection patterns and mediating variables for risk-injecting.

Results: Workshops designed for improving injection practices were associated with significant reductions in risk-injecting behaviors and a significant increase in using a new syringe. They were also associated with significant improvements in mediating variables for injection patterns: knowledge on injection-risk and AIDS, self-efficacy and motivation for risk-reduction in injecting drug use. Moreover, users of these workshops reported high satisfaction levels.

Conclusions: The findings provide encouraging support for these short-interventions designed for risk-reduction among injecting drug users, and also for previous efforts in training a network of health professionals involved in risk reduction among drug users.

Key words: Harm Reduction, IDUs, Tailored Program, Workshop, AIDS

\section{INTRODUCCIÓN}

$\mathbf{E}$ n nuestro país, la creación y organización de los servicios de atención a las drogodependencias se produce a partir de 1975, teniendo como marco referente un modelo de la adicción como enfermedad que sustituyó al modelo imperante durante los años anteriores, en el que el usuario de drogas era considerado "peligroso social" y delincuente. Estos dos modelos de acercamiento a las drogodependencias, si bien difieren significativamente en su visión del sujeto consumidor de drogas, comparten el objetivo final a conseguir: el cese del consumo, bien a través de una "sociedad libre de drogas" bien a través de la abstinencia del sujeto.

Así, la gran mayoría de los programas asistenciales para los usuarios de drogas se han basado en la abstinencia y han dado prioridad a ésta frente a cualquier otro tipo de salida para el sujeto.

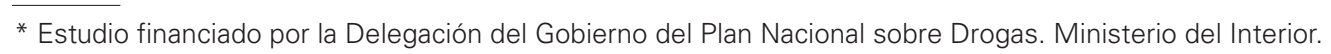


A partir de los años 90, y frente a la realidad de la epidemia de SIDA y al alcance de la infección por el Virus de la Inmunodeficiencia Humana (VIH) entre los inyectores de drogas (asociada, entre otros factores, a la alta incidencia de uso inyectado de heroína a finales de los 70 cuando la gran mayoría de los usuarios de drogas no era consciente de los riesgos que se asumían al compartir los materiales de inyección) y a la inexistencia de un tratamiento eficaz para esta infección, los esfuerzos sanitarios se comenzaron a centrar en la prevención, observándose en distintos países de Europa una tendencia hacia el abandono de los objetivos de abstinencia y hacia la adopción de modelos de reducción de los riesgos asociados al uso de drogas inyectadas.

No obstante, no todos los países adoptaron las políticas de reducción de riesgos, muchos se posicionaron claramente en contra y otros comenzaron a considerarlas, aunque con muchas precauciones y temores.

La crítica principal a los Programas de reducción de los riesgos asociados al consumo de drogas ha sido el cuestionamiento de su eficacia, y las consecuencias derivadas de objetivos que no persiguen la abstinencia. Sin embargo, numerosos estudios han demostrado ya su efectividad y eficiencia, su carácter no excluyente con otros Programas de tratamiento para las adicciones y la necesidad ineludible de que complementen a Programas destinados a conseguir la abstinencia.

En España, ante la falta de una política unificada de actuación frente al uso de drogas los distintos dispositivos de tratamiento (públicos y privados) decidieron aisladamente que tipo de Programas poner en marcha, coincidiendo en general en Programas orientados a la abstinencia. Sin embargo, en los últimos años, se ha producido en las distintas CC.AA. y en las organizaciones que trabajan con usuarios de drogas, un acercamiento hacia la necesidad de diseñar e implementar Programas de prevención de los problemas de salud que se enmarquen en los modelos de Reducción de Riesgos con los usuarios de drogas (especialmente con aquellos que las utilizan por vía parenteral, aunque como bien sabemos es difícil categorizar a las personas como "usuario de drogas inyectadas" -UDI- vs. No UDI de manera excluyente, ya que muchas veces se está en la frontera y, dependiendo de distintos factores se realiza o no la conducta de inyección). ${ }^{1}$

Algunas premisas básicas del modelo de reducción de riesgos son (Marlatt, 1998):

a) Se acepta la evidencia de que las personas continuarán consumiendo drogas, de que no todos los consumidores de drogas están en condiciones de rea- lizar un tratamiento de desintoxicación (porque no pueden o no quieren) y de que muchos de los que consumen no se acercan ni contactan con los servicios sanitarios (existiendo un período de latencia prolongada entre el consumo regular de una sustancia y por ende, de los riesgos y daños asociados, y la demanda de atención en los centros de tratamiento). En esta línea, podríamos definir el uso de drogas como un fenómeno complejo y multicausal, que supone un "continuum" desde la abstinencia hasta la dependencia, desde la ausencia de problemas hasta los riesgos y daños más graves y vitales; lo que conlleva concebir las intervenciones circularmente y ampliarlas a todos los momentos del proceso.

b) Debe tenerse en cuenta que los riesgos derivados del consumo de drogas son diversos y dependen de diferentes factores como son: el tipo de droga consumida, la frecuencia y la cantidad, cómo se administra, las circunstancias físicas y sociales de este consumo, y las políticas sociales para reducirlo. Es importante señalar que en algunos casos las políticas para reducir este consumo pueden aumentar el riesgo asociado con el uso de drogas, como cuando sólo se ofrecen servicios dirigidos a la abstinencia. La reducción de riesgos si bien es compatible con la creencia de que cada uno tiene el derecho de consumir drogas si lo quiere, reconoce que la mayoría de las drogas producen dependencia fisiológica y/o psicológica y que el consumo de drogas perjudica la salud.

c) Muchas veces, los problemas asociados al uso de drogas, se deben más a los hábitos y patrones de consumo que a los efectos de las drogas en sí mismas, no siendo tan importante qué se consume sino cómo se consume. Así, muchos de los riesgos relacionados con las drogas pueden ser eliminados con éxito sin reducir necesariamente el consumo de éstas.

d) Los daños asociados al consumo de drogas son multidimensionales. El receptor del daño puede ser el propio individuo, su contexto grupal próximo (familia, amigos, vecinos) o la comunidad en general, por tanto, las estrategias a poner en marcha para disminuir los daños deben tener en cuenta distintos niveles: individual, grupal, social y político.

e) La reducción de riesgos no se plantea como una medida opuesta a la abstinencia, sino como complementaria y facilitadora de éste y de otros objetivos a medio y a largo plazo.

f) Se promueve la competencia y responsabilidad de los propios consumidores de drogas, incluyendo, pero no limitándose al consumo de sustancias. Para ello se solicita la opinión de los propios consumidores en el diseño de las políticas y programas creados para

1 Teniendo en cuenta esta realidad, nos vamos a referir a usuarios de drogas inyectadas -UDIs-, incluyendo entre estos a aquellos usuarios esporádicos de la vía parenteral, ya que ellos también están realizando conductas de riesgo para la transmisión del VIH y otros agentes patógenos. 
responder a sus necesidades y se promueve su participación activa en los mismos, potenciando su formación como agentes de salud.

Distintas razones justifican la necesidad de poner en marcha en los servicios de atención a las drogodependencias este tipo de Programas: a) la alta tasa de seroprevalencia frente al VIH, VHB y VHC en este colectivo; b) el que la mayoría de los UDIs realicen los dos comportamientos específicos que explican la mayor parte de las infecciones por VIH en adultos (vgr. compartir jeringuillas infectadas y tener relaciones sexuales coitales sin preservativo); c) el que una proporción importante de las mujeres UDIs está en edad de procrear y raramente practica una contracepción sistemática con el consiguiente riesgo de transmisión materno-fetal del VIH; d) el que en el colectivo de mujeres UDIs la práctica de la prostitución es más frecuente que en hombres UDIs y la pareja estable de estas mujeres es, en numerosas ocasiones, también usuaria de drogas, siendo estas parejas sexuales de las mujeres UDIs las que ejercen la mayor influencia a la hora de compartir el material de inyección y de realizar comportamientos de riesgo para la transmisión del VIH (Donoghoe et al., 1989; Murphy, 1989; Kline y Stricker, 1993).

Teniendo en cuenta esta filosofía de trabajo, conociendo la realidad de la infección por VIH, VHB y VHC en el colectivo de UDIs en nuestro país (que presentaba la tasa de incidencia anual de sida más elevada de Europa) y conociendo también la importancia que tiene la formación de los profesionales sanitarios, para la puesta en marcha de programas eficaces de reducción de riesgos con colectivos específicos, (Mantell, DiVittis y Auerbach, 1997; Marlatt, 1998; Gibney, DiClemente y Vermund, 1999), el Plan Nacional sobre Drogas a través de un convenio con el Plan Nacional sobre el Sida y la Universidad del País Vasco ponen en marcha un Programa en Redes para la formación de profesionales sanitarios que trabajan en Servicios Públicos de atención a UDIs (Insúa y Moncada, 2000a; 2001). Este Programa tiene en cuenta los principios de las intervenciones eficaces de Salud Pública; las que, además de ofrecer información proporcionan a los individuos los medios para cambiar sus conductas; es decir, además de aumentar la conciencia sobre los riesgos de salud y los métodos eficaces para reducirlos, proporcionan tanto medios prácticos para el cambio de conducta (agujas, condones, jeringuillas, tratamiento para su adicción, etc.) como la ayuda necesaria para desarrollar habilidades personales de cara a posibilitar la reducción de los riesgos y el cambio conductual). El diseño de la formación y las intervenciones se ha realizado y considerando los progra- mas cuya evaluación ha demostrado que son eficaces para los objetivos que se proponen.

Este Programa, tiene sus fuentes teóricas en los Modelos sociocognitivos que explican el cambio de comportamiento (el Modelo de la Acción Razonada (Ajzen y Fishbein, 1977; 1980); el Modelo de la Acción Planificada (Ajzen y Madden, 1986; Shifter y Ajzen, 1985); el Modelo de Creencias de Salud (Becker, 1974); el Modelo de Prevención de Recaídas (Gibbons, McGobern y Lando, 1991); el Modelo PRECEDE-PROCEED (Green y Kreuter, 1991); la Teoría de la Autoeficacia (Bandura, 1977); el Modelo de Fases de Cambio (Prochaska y DiClemente, 1983; 1992); el Modelo de Reducción de Riesgo de Sida (Catania et al., 1990) y el Modelo de Reducción de Riesgo de Sida Modificado (Ehrhardt et al., 1992)) y se consolida después de haber sido puesto a prueba y evaluado en distintas poblaciones de profesionales sanitarios del Estado español durante los últimos años.

Estas evaluaciones han permitido estructurar un Programa de intervención en Redes que se concibe como una unidad que incluye formación en dos tipos de talleres: Talleres de sexo más seguro (TSMS) y de consumo de menos riesgo (TCMR), supervisión para el diseño de intervenciones adaptadas a cada territorio y un Manual de Educación Sanitaria y dos vídeos psicoeducativos (Insúa, 1999) como materiales de apoyo.

El Programa se realizó en cuatro fases: a) En la primera fase se contactó con los responsables de los Planes de Drogas de las Comunidades Autónomas y de distintas ONGs de carácter supracomunitario. Esta primera fase contemplaba también el diseño del Programa de Formación de Formadores. Como resultado de esta primera fase fueron formados 56 profesionales de las distintas CC.AA.; b) Durante la segunda fase los profesionales formados diseñaron Talleres adaptados a su realidad laboral ${ }^{2}$. En total fueron diseñados 21 TCMRs y 22 TSMSs. Además, estos profesionales revisaron el material e hicieron aportaciones que fueron recogidas en la edición final del Manual de Educación Sanitaria; c)La tercera fase consistió en la realización de Programas de Prevención de la Transmisión del VIH por parte de estos profesionales formados, con profesionales sanitarios de las redes públicas de asistencia a las drogodependencias de sus respectivas CC.AA. A cada territorio se asignó un determinado numero de cursos -entre uno y tres- en función de su población y del número de admisiones a tratamiento. Estos cursos fueron apoyados con financiación y materiales. Como resultado de esta fase de aplicación, se pudo multiplicar por 12 en un año, el número de profesionales formados en este tipo de Programas, ya que se realizaron un total de 41 Talleres que forma-

2 Se supervisó el diseño tanto de los TSMSs como de los TCMRs realizados por los profesionales formados en los Cursos, de tal manera que estos Talleres cumplieran los criterios científicos y metodológicos necesarios para conseguir los objetivos de formación que se pretendía. 
ron a 676 profesionales; d) Por último la cuarta fase supuso la realización de estos Programas de Prevención con distintos grupos de usuarios de drogas del Estado (Insúa y Moncada, 2000a, 2001).

El Programa de Formación de Formadores, trabajó especialmente en el cambio en las propias actitudes y creencias de los profesionales con respecto al consumo de drogas y a la conducta sexual y les capacitó para conceptualizar, diseñar, implementar, y evaluar Programas de reducción de los riesgos asociados al consumo de sustancias, en la forma de TSMS y TCMR.

Nuestra idea fue crear una red de trabajo en el tema de la prevención de la transmisión del VIH con usuarios de drogas, en la que los profesionales de distintas provincias se conocieran e intercambiaran experiencias, diseñaran Programas de intervención eficaces con características comunes (aquellas que se han demostrado útiles en los programas pilotos) y los evaluaran con los mismos instrumentos (para poder así compartir los resultados, comparar poblaciones, etc.).

\section{MATERIAL Y MÉTODO}

\subsection{Descripción del Programa de intervención con usuarios de drogas $\left(4^{\mathrm{a}}\right.$ fase del Programa de For- mación de Formadores)}

El objetivo general de este Programa de intervención es la reducción de riesgos y daños asociados al consumo de drogas entre usuarios de drogas admitidos en centros de asistencia y tratamiento de todo el Estado.

Se desarrollaron actividades de formación para la prevención de los problemas de salud asociados al consumo de drogas y específicamente para la prevención de la infección y problemas relacionados con el $\mathrm{VIH}$ y SIDA. Estas actividades estuvieron organizadas en forma de TCMRs y TSMSs, siguiendo los criterios de las intervenciones eficaces de Salud Pública recogidos en otros trabajos nuestros (Insúa y Moncada, 2000b; Insúa, Lledó y Grijalvo, 2001) y teniendo como objetivo la reducción de las conductas de riesgo (tanto de inyección como sexuales) asociadas al consumo y no el cese o la reducción del consumo de sustancias en sí mismo.

En este trabajo presentamos exclusivamente los resultados de los TCMRs para usuarios de drogas diseñados, implementados y evaluados por los profesionales de las CC.AA. del Estado que habían sido formados previamente en nuestro Programa de Formación de Formadores.

Los TCMRs fueron diseñados y realizados por profesionales sanitarios de 16 CC.AA. del Estado español, con la participación de una ONG. Para el estudio que presentamos, hemos escogido una submuestra de 15 Talleres cuyos conductores aceptaron entrar en un riguroso protocolo de evaluación y en total han participado 229 usuarios de drogas inyectadas en tratamiento en centros de atención a las drogodependencias. Los Talleres se realizaron durante el periodo Enero - Agosto del año 2001.

\subsubsection{Fases en el desarrollo del Programa de interven- ción con usuarios de drogas}

Para la realización del Programa de intervención con usuarios de drogas, se siguieron distintas fases: 1) Reunión con los Coordinadores de las diferentes CC.AA. participantes en el Programa global y especificación de los objetivos y metodología de los talleres para UDIs; 2) Selección de los Monitores responsables de la implementación de los Talleres en cada CC.AA., de entre los profesionales formados en el Programa de Formación de Formadores; 3) Diseño de Ios TCMRs para UDIs; 4) Supervisión de los TCMRs diseñados, por el equipo docente del Programa de Formación de Formadores; 5) Organización de la evaluación del Programa con UDIs y 6) Puesta en marcha del Programa, implementación de los TCMRs y evaluación de los mismos.

\subsubsection{Metodología utilizada para el desarrollo de los Talleres}

El desarrollo de los Talleres se basó en una metodología que combinó técnicas grupales para la participación de los usuarios, la exposición semi-magistral activa de contenidos teóricos y el uso de técnicas directivas y de dinámicas de grupo para la organización de las sesiones. La duración de los Talleres fue de 10 horas, distribuidas en cinco sesiones de dos horas cada una.

\subsubsection{Objetivos de los TCMRs}

Los objetivos generales de los TCMRs son: a) Aumentar la información sobre enfermedades infectocontagiosas; b) Disminuir conductas de riesgo en el uso de drogas; c) Reducir las infecciones y demás problemas médicos asociados al consumo de drogas.

Los objetivos específicos de los TCMRs son: a) Favorecer la identificación de un consumo de drogas que conlleva riesgos para la salud; b) Aumentar la percepción de los riesgos y daños para la salud asociados a las prácticas de consumo de drogas; c) Aumentar la autoeficacia personal frente a situaciones de riesgo para el consumo de drogas; d) Promover el aprendizaje y práctica de habilidades personales y sociales necesarias para adoptar un patrón de consumo de drogas de menor riesgo; e) Aumentar los conocimientos para identificar y responder eficazmente ante una sobredosis por consumo de drogas. 


\subsection{Plan de eEvaluación del Programa de Interven- ción con UDIs.}

\subsubsection{Objetivos de la evaluación}

La evaluación de los Talleres se centra en dos ámbitos de evaluación:

- Evaluación de los Resultados e Impacto de los Talleres (Eficacia): que busca evaluar la evolución de las conductas de consumo de drogas que constituyen un riesgo para la salud y evaluar el cambio en las variables mediadoras que explican el cambio en las conductas de riesgo (conocimientos, actitudes, expectativas, percepción del riesgo y control).

- Evaluación de los Procesos, cuyo objetivo es la valoración de la adaptación del proceso de implementación de los Talleres a lo planificado y la valoración de la percepción satisfacción de los usuarios de los Talleres acerca de los diferentes elementos estructurales del Taller, impacto y satisfacción global.

El propósito es evaluar los resultados y procesos de los Talleres, comparando (en la evaluación de los resultados) los usuarios de los Talleres con sujetos en tratamiento que no han participado en los mismos.

\subsubsection{Diseño de la evaluación}

El diseño de la evaluación del Programa de intervención es de tipo cuasi-experimental $2 \times 3$, con medidas repetidas y un grupo de comparación. Los sujetos fueron asignados de forma no aleatoria a una de las dos condiciones (usuarios de los Talleres o grupo de comparación).

Los momentos de medida fueron tres:

- Evaluación basal (pretest), durante el primer día de implementación de los Talleres.

- Evaluación postest, inmediatamente tras la finalización de los Talleres.

- Evaluación de seguimiento, un mes después de la finalización de los Talleres.

\subsubsection{Variables e instrumentos de medida}

El instrumento utilizado para la recogida de información fue un cuestionario estructurado con preguntas cerradas agrupadas en los siguientes conceptos: a) Datos sociodemográficos (12 ítems); b) Consumo de drogas: sustancias, frecuencia y vía de consumo (34 ítems); c) Sobredosis (20 ítems); d) Momento personal de cambio en la conducta de inyección de drogas ( 7 ítems); e) Conocimientos sobre el SIDA (30 ítems); f) Conocimientos sobre riesgos asociados a la inyección de drogas (29 ítems); g) Percepción del riesgo asociado a la situación (8 ítems) y h) Conductas de inyección (22 ítems). Todos los instrumentos utilizados para la recogida de los datos expuestos en el presente informe han demostrado adecuadas consistencia interna y fiabilidad test - retest en su aplicación a los usuarios de los TCMR (Insúa, 2002).

\subsubsection{Análisis estadísticos utilizados}

En este trabajo se han utilizado los siguientes análisis estadísticos, realizados con el paquete estadístico SPSS, v.10.1.

- Análisis descriptivos: Frecuencias y porcentajes, estimadores clásicos de localización y de escala (media, desviación típica, etc).

- Análisis comparativos: Tablas de contingencia y estadísticos asociados; comparación de medias basadas en la prueba t para muestras relacionadas (en la comparación intra-grupo a lo largo del estudio) y para muestras independientes (comparación inter-grupos en cada uno de los momentos de medida); análisis de covarianza; pruebas no paramétricas para la comparación intra grupo e inter grupos (la prueba de McNemar) y análisis de varianza de medidas repetidas.

\subsubsection{Composición de la muestra.}

La muestra participante en el estudio para la evaluación de los Talleres de consumo de menor riesgo estaba formada por 229 sujetos, 157 hombres $(68,6 \%)$ y 72 mujeres $(31,4 \%)$, procedentes de programas de tratamiento de 10 Comunidades Autónomas (Andalucía, Aragón, Cantabria, Castilla y León, C. Valenciana, Extremadura, Rioja, Madrid, Murcia y Navarra). Estos programas de tratamiento eran en su mayor parte programas ambulatorios de mantenimiento con metadona (PMM), y algún programa de intercambio de jeringuillas (PIJ). La mayoría de los sujetos son de nacionalidad española (91,5\%). La media de edad es de casi 34 años (D.E. de 6 años). Algo más de la mitad están solteros $(54,6 \%)$, alrededor de la cuarta parte están casados o con pareja estable $(26,2 \%)$ y poco menos de la mitad tienen hijos $(43,1 \%)$. La mayoría de ellos tienen estudios equivalentes a la EGB o inferiores $(63,8 \%$ ) y la mayoría de ellos están sin empleo o en situación de incapacidad laboral (67\%). Más de la mitad han estado en prisión $(58,1 \%)$ y algo menos de la mitad son seropositivos al $\mathrm{VIH}(40,5 \%)$

Casi todos ellos han consumido alguna vez heroína $(97,3 \%)$, cocaína $(96,4 \%)$, speedball $(91,4 \%)$ y metadona $(83,1 \%)$. Más de la mitad han consumido en los últimos 3 meses metadona (78,6\%) y / o cocaína $(50,7 \%)$ y casi la mitad han consumido en esos últimos 3 meses speedball $(46,7 \%)$ y / o heroína sola $(36 \%)$. Casi todos se habían inyectado drogas alguna vez en su vida (94\%) y más de la mitad se habían inyectado drogas durante el último año (57,2\%).

De los 229 sujetos que participaban en el estudio para la evaluación de los 15 TCMRs, aproximadamente la mitad eran usuarios de los Talleres $(N=113$; 
$49,3 \%$ ) y componían por tanto el grupo de intervención; y los demás formaban parte de los grupos de comparación ( $\mathrm{N}=116 ; 50,7 \%)$.

\section{RESULTADOS}

Presentamos la información relativa a la evaluación de los resultados de los Talleres y la evaluación de la satisfacción de los usuarios con los mismos.

La evaluación de los resultados incluye información relativa a los principales indicadores de las conductas de riesgo de consumo inyectado de drogas. También incluye información sobre algunas de las variables mediadoras objeto de intervención en los Talleres. Ambos tipos de variables (variables mediadoras e indicadores de conductas de riesgo para la transmisión del VIH/SIDA) son descritas atendiendo a su evolución a lo largo del estudio (en el pretest, postest y seguimiento), comparando a los usuarios de los TCMRs con sus grupos de comparación.

La evaluación de la satisfacción de los usuarios incluye los resultados obtenidos en el postest y en el seguimiento de los TCMRs. De los 229 sujetos que participaban en el estudio para la evaluación de los 15 TCMRs, aproximadamente la mitad eran usuarios de los Talleres ( $\mathrm{N}=113,49,3 \%$ ) y componían por tanto el grupo de intervención; y los demás formaban parte de los grupos de comparación ( $\mathrm{N}=116,50,7 \%)$.

\subsection{Evaluación de resultados de los TCMR:}

Los indicadores conductuales utilizados como medidas de resultados de los TCMRs en el presente trabajo son: a) Utilización siempre de jeringuilla nueva para la inyección de drogas; b) Reutilización de la jerin- guilla propia para la inyección de drogas; c) Reutilización de la jeringuilla de otra persona para la inyección de drogas; d) Compartir otros materiales de inyección; e) Pasar la propia jeringuilla a otra persona.

Las variables mediadoras utilizadas como medidas de resultados de los TCMR son: a) Conocimientos sobre la inyección de riesgo, y b) Conocimientos sobre el SIDA y c) Otras variables mediadoras en la inyección de riesgo: momento personal hacia el cambio y expectativas de asumir riesgos en la inyección de drogas ante diferentes situaciones.

\subsubsection{Utilización siempre de una jeringuilla nueva para la inyección de drogas}

Apenas uno de cada cinco de los usuarios de los TCMRs utilizaban siempre jeringuilla nueva para inyectarse drogas en los tres meses anteriores al pretest. Al compararlos con los sujetos del grupo de comparación, las diferencias no eran estadísticamente significativas (Chi cuadrado $=2,5 ; 1$ g.l.; $p<0,16$ ), indicando una homogeneidad entre los sujetos de cara a la realización de esta conducta.

Tras los Talleres, más de la mitad de los usuarios de los TCMRs habían utilizado siempre jeringuilla nueva cada vez que se habían inyectado (durante los Talleres), el doble del porcentaje observado entre los sujetos de comparación. Las diferencias en el postest son estadísticamente significativas a favor de los usuarios de los TCMRs (Chi cuadrado = 4,5, 1 g.l.; $p<0,031$ ).

El porcentaje de usuarios de los TCMRs que utilizaron siempre jeringuilla nueva durante el seguimiento al mes posterior a los TCMRs es menor que tras los Talleres. Así, tras el seguimiento, las diferencias entre el grupo de intervención y el grupo de comparación no son ya estadísticamente significativas, aunque el porcentaje entre los usuarios de los TCMRs es el doble

\section{Gráfico 1. Comparación entre los dos grupos con respecto al uso siempre de una jeringuilla nueva (\%)}

\section{INTERVENCIÓN - -COMPARACIÓN}

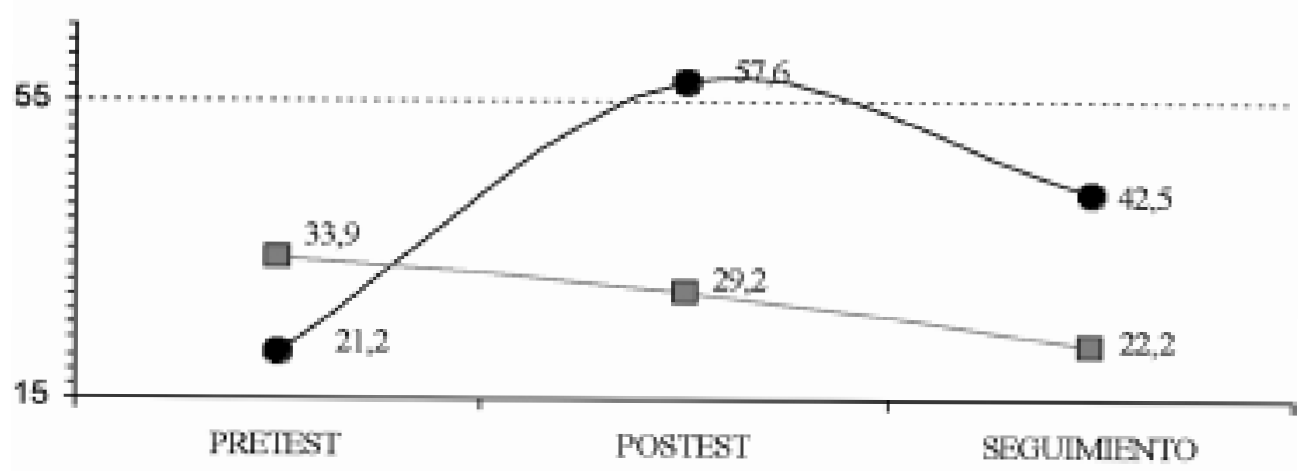


que entre los sujetos de comparación (Chi cuadrado = 2,9, 1 g.l.; $p<0,072$ ).

Entre los usuarios de los TCMRs, el porcentaje de uso siempre de jeringuilla nueva aumenta significativamente entre el pretest y el postest según la prueba de McNemar ( $p<0,000)$, y también entre el pretest y el seguimiento ( $p<0,011)$. Sin embargo, entre los sujetos de comparación no hay cambios significativos entre el pretest y el postest $(p<0,5)$ ni entre el pretest y el seguimiento $p<0,63$ ).

En resumen, los TCMR van asociados a un aumento significativo de la probabilidad de usar siempre jeringuilla nueva entre sus usuarios, y no es así entre los sujetos del grupo de comparación (ver gráfico 1).

\subsubsection{Reutilización de la propia jeringuilla para la inyec-} ción de drogas

Durante los tres meses anteriores al pretest habían reutilizado su propia jeringuilla aproximadamente tres de cada cuatro usuarios de TCMRs, frente a la mitad de los sujetos del grupo de comparación, con diferencias estadísticamente significativas (Chi cuadrado $=7,1,1$ g.l.; $p<0,006$ ).

En el postest habían reutilizado su propia jeringuilla menos de la mitad de los usuarios de los TCMRs y $2 / 3$ de los sujetos de comparación. Estas diferencias muestran una tendencia hacia la significatividad estadística (Chi cuadrado = 3,3, 1 g.l.; $p<0,061$ ). Durante el seguimiento habían reutilizado su propia jeringuilla más de la mitad de los usuarios de los TCMR y tres de cada cuatro de los sujetos del grupo de comparación, sin diferencias significativas (Chi cuadrado $=2,6,1$ g.l.; $p<0,081$ ).

En comparación con el pretest, los usuarios de los TCMRs disminuyeron significativamente la reutilización de la propia jeringuilla en el postest $(p<0,000)$ y en el seguimiento ( $p<0,006$ ) según la prueba de McNemar. Sin embargo, entre los sujetos de comparación no hay cambios en relación con el pretest ni en el postest $(p<0,69)$ ni en el seguimiento $(p<0,19)$.

En resumen, nuevamente se observa que la participación en los TCMRs va asociada a una disminución significativa de la conducta de riesgo de reutilización de la propia jeringuilla entre los sujetos que participan en ellos que no se da entre los sujetos del grupo de comparación (ver gráfico 2).

\subsubsection{Reutilización de la jeringuilla de otra persona} para la inyección de drogas

Con respecto a la reutilización de la jeringuilla de otra persona durante los tres meses anteriores al pretest, no había diferencias entre ambos grupos (Chi cuadrado $=0,28,1$ g.l.; $p<0,61$ ).

En el postest, ninguno de los usuarios de los TCMRs habían reutilizado la jeringuilla de otro, mientras que un pequeño número de los sujetos de comparación seguían haciéndolo. Las diferencias entre ambos grupos siguen sin ser estadísticamente significativas en el postest (Chi cuadrado $=2,9,1$ g.l., $p<$ $0,17)$. En el seguimiento, solamente 2 sujetos $(5 \%)$ de los usuarios de los TCMRs habían reutilizado la jeringuilla de otro, mientras que sí lo habían hecho el 25\% de los sujetos del grupo de comparación. Las diferencias entre grupos en el seguimiento son estadísticamente significativas (Chi cuadrado $=4,8,1$ g.l.; $p<$ 0,036).

En relación con el pretest, los usuarios de los TCMRs disminuyen significativamente la probabilidad de haber usado la jeringuilla de otro según la prueba de McNemar, tanto en el postest ( $p<0,008$ ) como en el seguimiento $(p<0,008)$. Sin embargo, entre los sujetos de comparación no se observan cambios sig-

\section{Gráfico 2. Comparación entre los dos grupos con respecto a la reutilización de su propia jeringuilla (\%)}

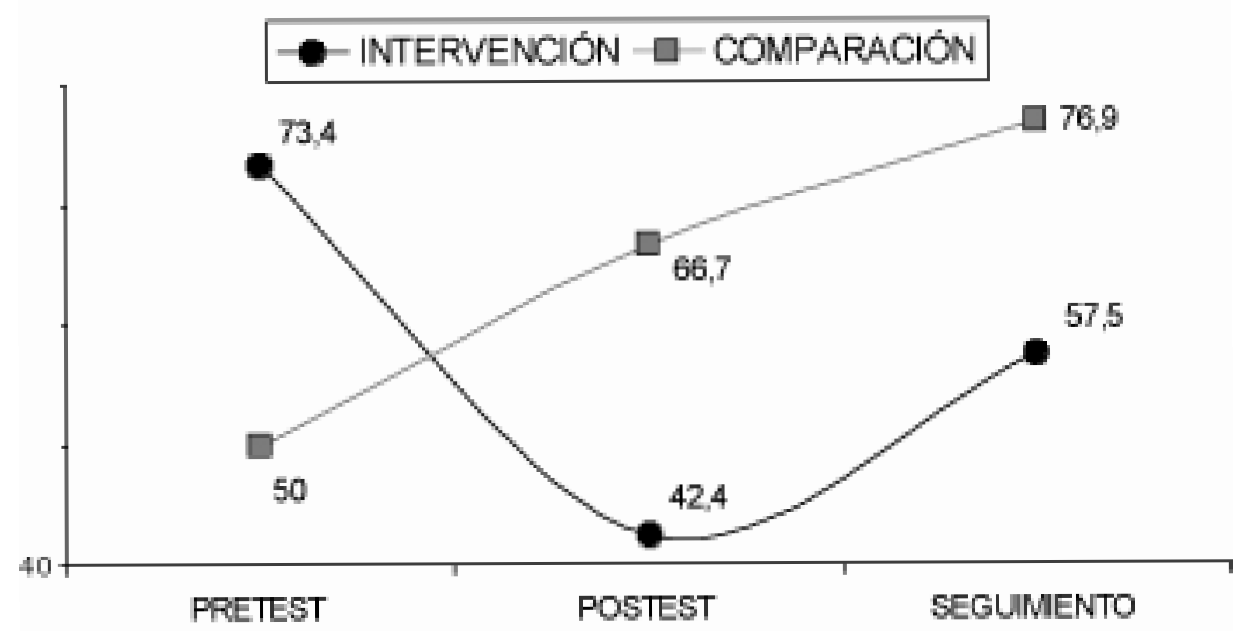




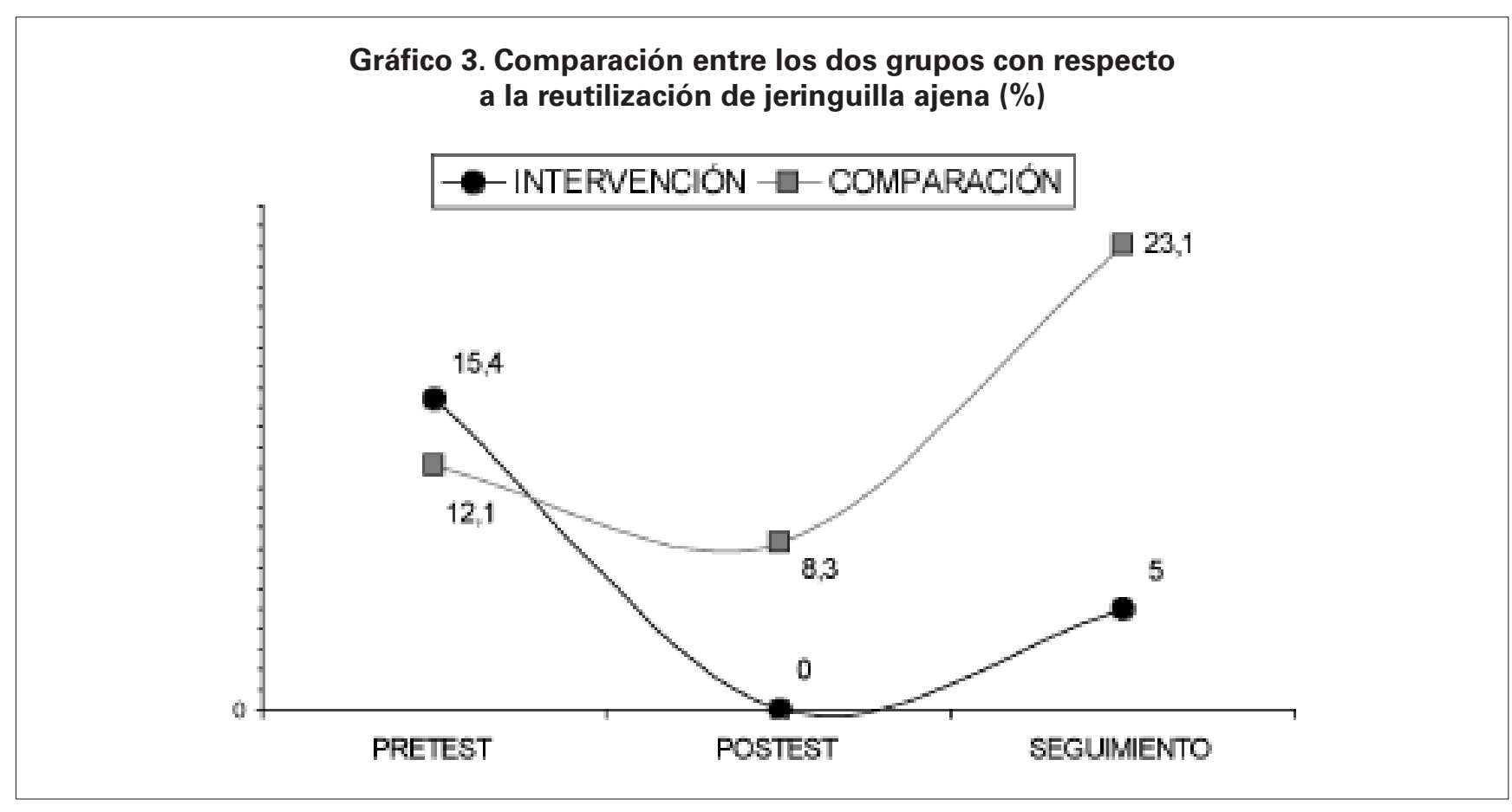

nificativos en esta conducta de riesgo ni en el postest ni en el seguimiento en comparación con el pretest.

Así pues, de nuevo se encuentra evidencia indicando que la participación en los TCMRs va asociada a una disminución significativa en la probabilidad de reutilizar la jeringuilla de otra persona, mientras que no se producen cambios entre los sujetos que no han participado en dichos Talleres (ver gráfico 3).

\subsubsection{Compartir el vaso para limpiar la jeringuilla}

De nuevo se observa que durante los tres meses previos al pretest los usuarios de los TCMRs habían realizado esta conducta de riesgo con mayor probabilidad que los usuarios de comparación (la probabilidad es 4 veces mayor, Chi cuadrado $=19,3,1$ g.l.; $p<$ 0,000). La mitad de los usuarios de los TCMRs habían compartido el vaso para limpiar la aguja y la jeringuilla.

Tras los Talleres, en el postest y en el seguimiento ya no hay diferencias significativas entre ambos grupos (Chi cuadrado $=0,1$ g.l. $p<0,62$ y Chi cuadrado $=0,58,1$ g.l.; $p<0,31$ respectivamente en el postest y seguimiento). Tras los TCMRs el $25 \%$ de los sujetos había compartido el vaso y en el seguimiento lo habían hecho entre un 30 y un $40 \%$.

Los sujetos que no participaron en los TCMRs no modificaron significativamente su probabilidad de compartir el vaso a lo largo del estudio en comparación con el pretest según la prueba de McNemar, ni en el postest $(p<0,5)$ ni en el seguimiento ( $p<$ 0,063).

Una vez más, los usuarios de los TCMRs disminuyeron significativamente la probabilidad de la conducta de riesgo en comparación con el periodo de tres meses previo al pretest, según la prueba de $\mathrm{McNe}$ mar. La probabilidad de haber compartido el vaso es significativamente menor en el postest y en el seguimiento que en el pretest ( $p<0,002$ y $p<0,001$ respectivamente). Se demuestra que la participación en los TCMRs también va asociada a una disminución de la conducta de compartir el vaso en comparación con la línea base, mientras que no se producen cambios entre quienes no participaron en los Talleres (ver gráfico 4).

\section{1.5. Pasar la propia jeringuilla a otra persona}

También la conducta de pasar la jeringuilla propia a otra persona era más frecuente en los tres meses anteriores al pretest entre los usuarios de los TCMRs que entre los sujetos de comparación, aunque las diferencias no son estadísticamente significativas (Chi cuadrado $=2,7,1$ g.l.; $p<0,14)$. Uno de cada cinco usuarios lo habían hecho, por uno de cada 10 sujetos de comparación.

Tampoco hay diferencias significativas entre ambos grupos ni en el postest (Chi cuadrado $=1,6,1$ g.l., $\mathrm{p}<0,19$ ) $\mathrm{ni}$ en el seguimiento (Chi cuadrado $=$ 1,9, 1 g.l., $p<0,15)$.

Tanto en el postest como en el seguimiento se invierte la relación observada en el pretest, de modo que la probabilidad de haber pasado la jeringuilla es doble entre los sujetos del grupo de comparación que entre los usuarios de los TCMRs.

Las reducciones observadas en la conducta de riesgo entre el postest y el pretest en los usuarios de los TCMRs no llegan a ser estadísticamente significativas según la prueba de McNemar $(p<0,11)$, aunque la reducción si es importante desde el punto de vista 
Gráfico 4. Comparación entre los dos grupos con respecto a compartir el vaso para limpiar la jeringuilla (\%)

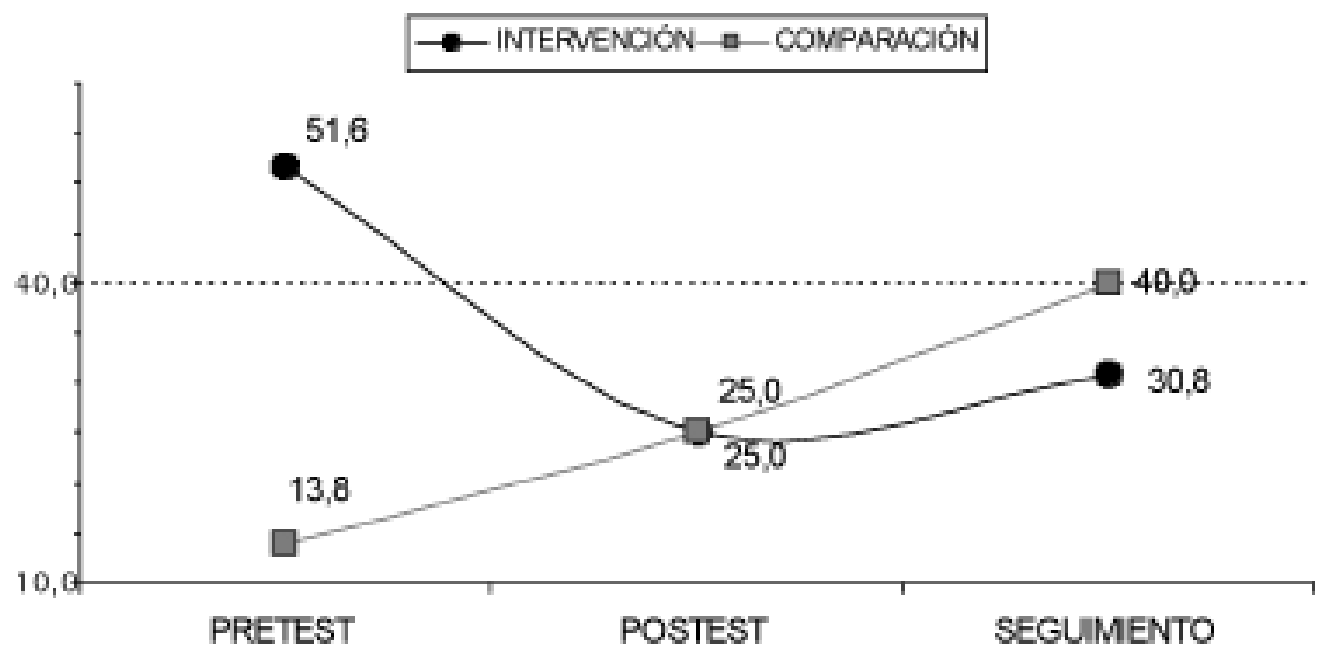

práctico. Tampoco se observan cambios estadísticamente significativos entre los sujetos de comparación a lo largo del estudio. Sin embargo, los análisis loglineales demuestran que la evolución observada en el postest en relación con el pretest es más favorable entre los usuarios de estos TCMRs que entre los sujetos de comparación (Chi cuadrado $=4,8 ; p=0,03$ ) y lo mismo ocurre en el seguimiento en relación con el pretest (Chi cuadrado $=4,3 ; p=0,04$ ).

En resumen, los usuarios de los TCMRs reducen sustancialmente la conducta de proporcionar su propia jeringuilla durante los Talleres con relación al pretest, aunque las diferencias no sean estadísticamente significativas y aunque esa reducción no parece man- tenerse tras el seguimiento. Los sujetos que no participan en los TCMRs, al igual que en otras conductas de riesgo, no reducen la frecuencia de proporcionar su jeringuilla tras los Talleres y en el seguimiento, en relación con el pretest y muestran una evolución en esta conducta de riesgo significativamente menos favorable que los usuarios de estos TCMRs a lo largo del estudio (ver gráfico 5).

\subsubsection{Conocimientos sobre la inyección de riesgo}

Ambos grupos (de intervención y de comparación) mostraban en el pretest un nivel de conocimientos muy similar acerca de los riesgos asociados a la inyección de drogas, respondiendo acertadamente a 17 de las 29 cuestiones (casi el 60\%).

\section{Gráfico 5. Comparación entre los dos grupos con respecto a prestar su jeringuilla (\%)}

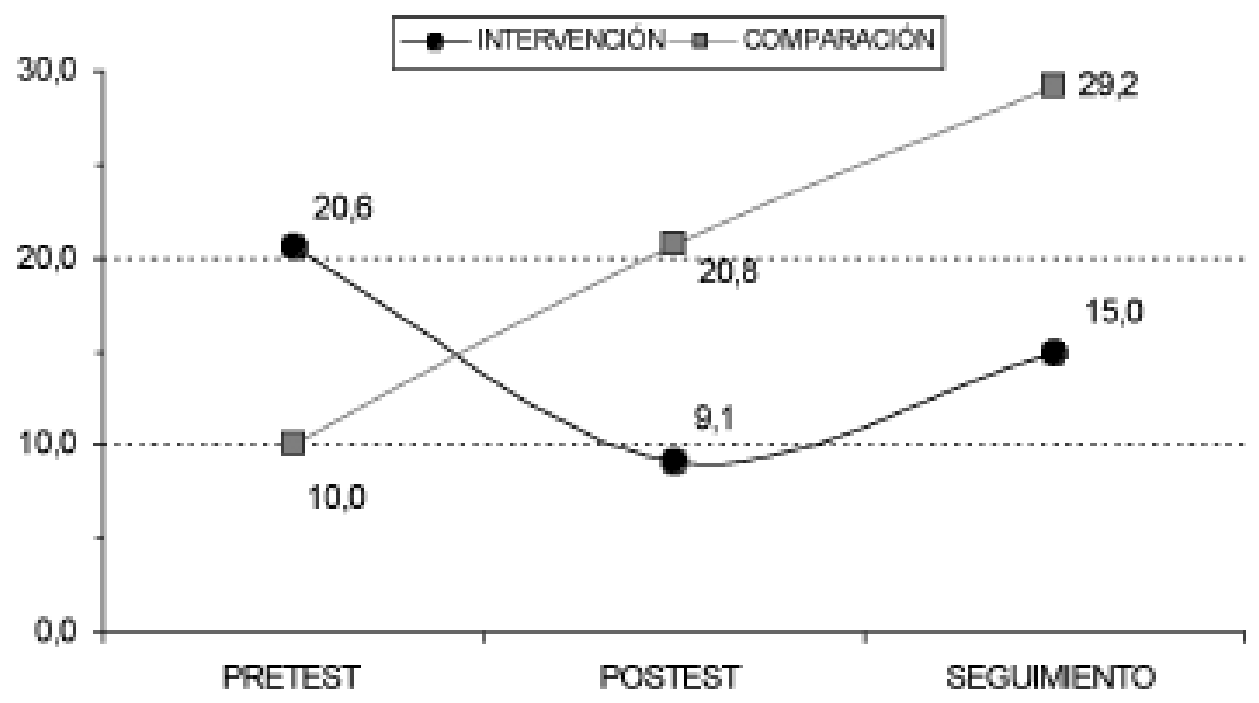


En el postest el nivel de conocimientos sobre la inyección de riesgo es significativamente superior entre los usuarios de los TCMR que entre el grupo de comparación, según la prueba T para la comparación de medias ( $t=6,212$ g.l.; $p<0,000$ ). Lo mismo ocurre en el seguimiento ( $t=5,3,211$ g.l.; $p<0,000$ ). Tanto en el postest como en el seguimiento los usuarios de los TCMR alcanzan una puntuación equivalente a responder acertadamente 22 de las 29 cuestiones sobre los riesgos de la inyección (el 75\% de las cuestiones), mientras que el grupo de comparación responde acertadamente al $63 \%$ de las mismas.

El análisis univariante de la varianza, tomando como covariante las puntuaciones en el pretest, demuestran que los usuarios de los TCMR incrementan sus conocimientos sobre la inyección de riesgo significativamente más que el grupo de comparación a lo largo del estudio en comparación con el pretest. Esto es así tanto en el postest ( $F=62,5,1$ g.l.; $p<$ $0,000)$ como en el seguimiento $(F=50,7,1$ g.l.; $p<$ $0,000)$. Los análisis de la varianza de medidas repetidas también demuestran que la evolución en el postest en relación con el pretest es más favorable entre los usuarios de los TCMR que entre los sujetos de comparación ( $F=59,9 ; 1$ g.l.; $p=0,000)$ y lo mismo ocurre en el seguimiento en relación con el pretest $(F$ $=49,4 ; 1$ g.l.; $p=0,000)$. Estos datos proporcionan apoyo a la eficacia de los TCMR para aumentar los conocimientos acerca de los riesgos asociados a la inyección de drogas (ver gráfico 6).

\subsubsection{Conocimientos sobre el SIDA}

El nivel de conocimientos sobre el SIDA era menor entre los usuarios de los TCMRs que entre los sujetos del grupo de comparación en el pretest, y pasó a ser superior en el postest y en el seguimiento. Según la prueba T para la comparación de medias esas diferencias no llegan a ser estadísticamente significativas en el pretest $\mathrm{ni}$ en el seguimiento $(\mathrm{t}=1,227 \mathrm{~g} . \mathrm{l}$.; $\mathrm{p}=$ 0,15 y $t=1,1,1$ g.l.; $p<0,13$ respectivamente). Sin embargo esas diferencias si son significativas en el postest, indicando mayor nivel de conocimientos sobre el SIDA entre los usuarios de los TCMR $(t=1,7$; 212 g.l.; $p<0,046)$.

Los análisis univariados de la varianza con las medidas del pretest como covariante demuestran que los usuarios de los TCMRs aumentan sus conocimientos sobre el SIDA tras los Talleres significativamente más que los sujetos de comparación ( $F=7 ; 1$ g.l.; $p<$ 0,009 ). Lo mismo ocurre con los cambios tras el seguimiento en relación con el pretest, que son significativamente más positivos entre los usuarios de los TCMRs que entre los sujetos de comparación $(F=5$; 1 g.l.; $p<0,027)$. Los análisis de la varianza de medidas repetidas también demuestran que la evolución en el postest en relación con el pretest es más favorable entre los usuarios de los TCMR que entre los sujetos de comparación $(F=7,7 ; 1$ g.l.; $p=0,006)$ y lo mismo ocurre en el seguimiento en relación con el pretest $(F=6,1 ; 1$ g.l.; $p=0,014)$.

Por tanto, estos resultados proporcionan apoyo a la eficacia de los TCMRs para aumentar los conocimientos sobre el SIDA entre sus usuarios (ver gráfico 7).

\subsubsection{Otras variables mediadoras en las conductas de} riesgo asociadas a la inyección de drogas.

Los usuarios de los TCMRs también evolucionaron durante los Talleres más positivamente que los sujetos de comparación en las otras dos variables mediadoras sometidas a estudio, según se demuestra con los análisis de varianza de medidas repetidas, aunque esa mejoría no se mantiene en el seguimiento. Los

\section{Gráfico 6. Comparación entre los dos grupos con respecto a los conocimientos sobre la Inyección de Riesgo (\%)}

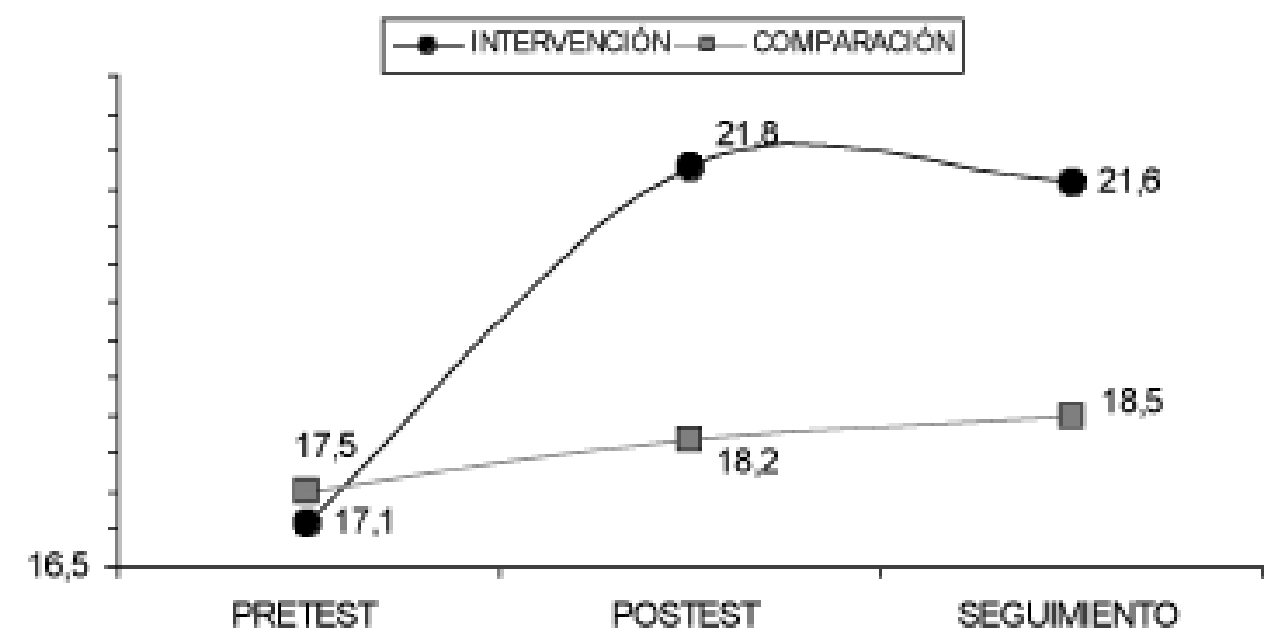




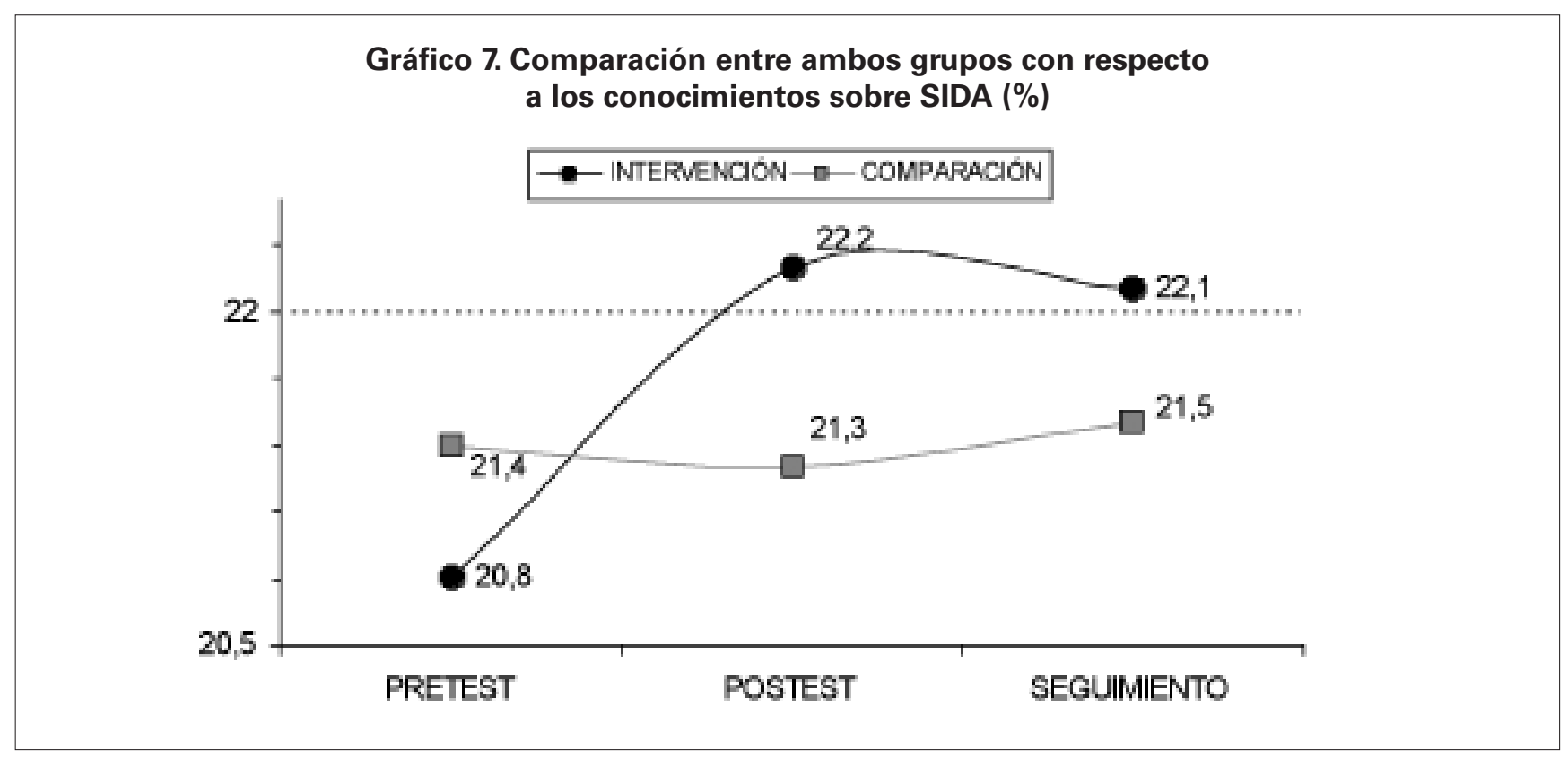

usuarios de los TCMRs redujeron más que los sujetos de comparación las expectativas de asumir riesgos en la inyección de drogas (tales como compartir jeringuillas, etc.) en el postest en relación con el pretest ( $F=$ 6,3; 1 g.I.; $p=0,012$ ). Los usuarios de los TCMRs también mantuvieron una alta motivación para la reducción de riesgos en la inyección de drogas en el postest, mientras que los sujetos de comparación redujeron significativamente esa motivación ( $F=5,5$; 1 g.l.; $p=0$ 02). Así pues, estos resultados también aportan evidencia de apoyo a favor de la eficacia de los TCMRs para modificar positivamente las expectativas de asumir riesgos en la inyección de drogas y para mantener una alta motivación hacia la reducción de estos riesgos.

\subsection{Evaluación de la Satisfacción de los usuarios con los TCMRs}

Cada uno de los 10 ítems del cuestionario de satisfacción se responde sobre una escala cuyos valores máximo y mínimo son 5 y 1 (mucho y nada satisfecho respectivamente).

La satisfacción del usuario está agrupada en tres dimensiones:

Satisfacción relativa a la adecuación del taller a las características del usuario: momento personal, interés suscitado, utilidad para el cambio en las conductas de riesgo y satisfacción personal.

Satisfacción relativa a aspectos específicos del taller: adecuación del local, metodología de exposición de los temas, adecuación del grupo e interés de este tipo de Talleres para un futuro.

Satisfacción con los recursos humanos del taller: competencia y accesibilidad del personal responsable de los Talleres.
3.2.1. Satisfacción relativa a la adecuación del taller a las características del usuario

Los usuarios consideran que los TCMRs se adecuan casi bastante bien a su momento personal idóneo, tanto en el postest como en el seguimiento.

Las puntuaciones relativas a la satisfacción con el taller en cuanto al interés suscitado en los usuarios, utilidad para el cambio en las conductas de riesgo y satisfacción personal están entre "bastante" y "máxima", tanto en el postest como en el seguimiento (ver gráfico 8).

3.2.2. Satisfacción del usuario con aspectos específicos del Taller

La satisfacción de los usuarios con el método para la exposición de los temas y el resto del grupo es notable, tanto en el postest como en el seguimiento.

La satisfacción con los locales es casi notable y el interés de que se realicen estos Talleres en el futuro es casi máximo, tanto en el postest como en el seguimiento (ver gráfico 9).

\subsubsection{Satisfacción de los usuarios con los recursos humanos del Taller}

La satisfacción de los usuarios de los TCMRs con los recursos humanos es entre notable y máxima, en competencia y accesibilidad, tanto en el postest como en el seguimiento (ver gráfico 10). 
Gráfico 8. Satisfacción de los usuarios de los TCMR. Componentes del usuario

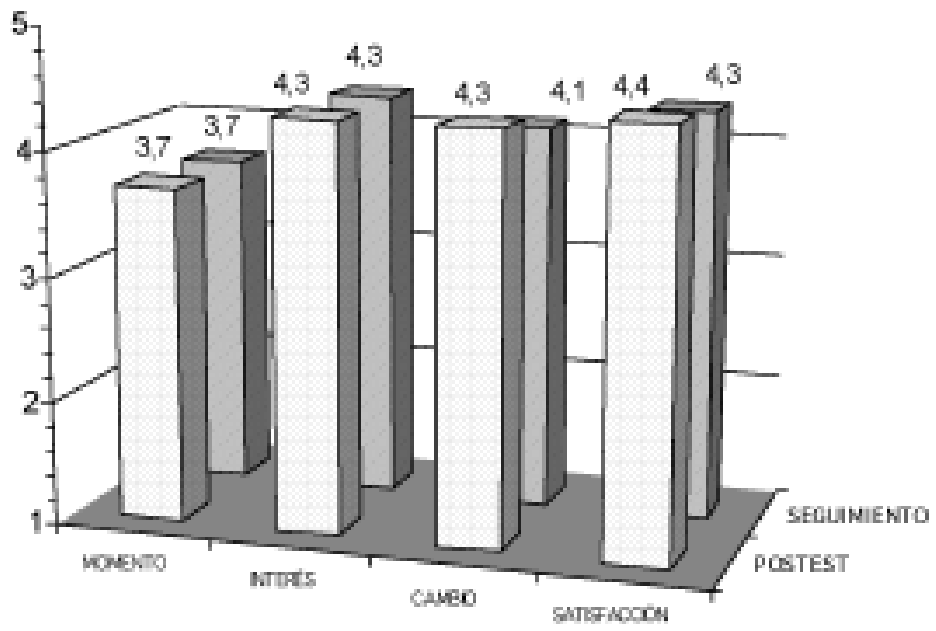

Gráfico 9. Satisfacción de los usuarios de los TCMR. Componentes del Taller

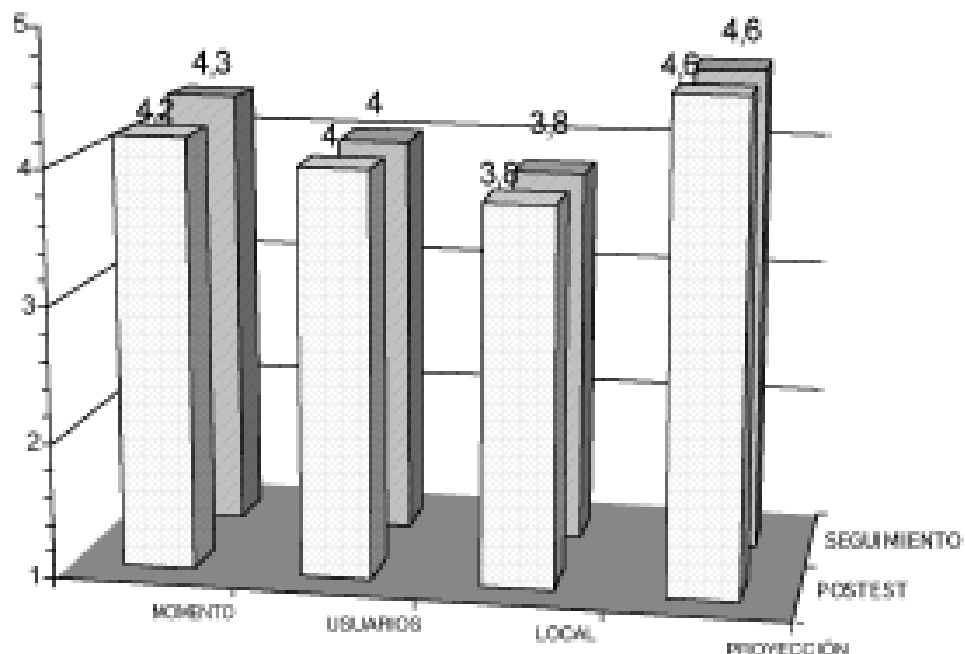

Gráfico 10. Satisfacción de los usuarios de los TCMR. Componentes del Personal Docente

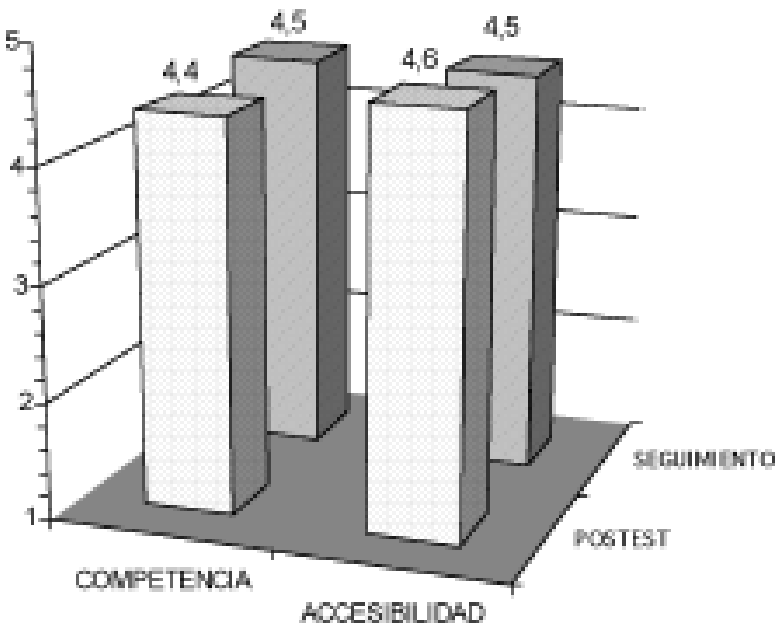




\section{DISCUSIÓNY CONCLUSIONES}

El primer resultado reseñable observado en nuestro estudio es que la frecuencia de las diferentes conductas de riesgo evaluadas en los últimos tres meses (inyectarse solo, usar jeringuilla usada: propia y / o ajena, compartir material de limpieza, proporcionar la jeringuilla a otro UDI) es mucho menor que la frecuencia de estas conductas en el ciclo vital de los sujetos evaluados. También hemos observado en el pretest de los pacientes en PMM o PIJ, altos niveles de motivación para la reducción de riesgos y alta autoeficacia para afrontar diversas situaciones que determinan la conducta de riesgo, reflejando actitudes positivas para la reducción de riesgos asociados al consumo inyectado de drogas. Numerosos estudios ya han demostrado que los UDIs son capaces de modificar sus conductas de riesgo asociadas al consumo inyectado de drogas para reducir estos riesgos (Watters, Estilo, Kral y cols., 1994; Watters, Estilo, Clark y Lorvick, J.,1994; Stimson y Power, 1992; Stimson, 1995). En resumen, tanto la mayoría de los estudios publicados como nuestros propios resultados vienen a demostrar que los programas de mantenimiento con metadona y los PIJs suelen disminuir sustancialmente el número y frecuencias de conductas de riesgo asociadas al consumo inyectado de drogas: consumo de drogas ilegales, inyección de drogas.

Así pues, se acepta que el tratamiento de los trastornos por dependencia a drogas, el contacto de los drogodependientes con instituciones de asistencia y tratamiento, y la disponibilidad de material y equipamiento de inyección estéril son condiciones a menudo necesarias para la reducción de riesgos para la salud asociados al consumo de drogas. Sin embargo, también hay diversos estudios que, al igual que el nuestro, demuestran que estas conductas de riesgo para la salud se mantienen en cierta medida en los PMM (Stark y cols., 1996; Grella, Anglin y Annon, 1996; Magura, Rosemblaum y Rodríguez, 1998; Des Jarlais, 1999) y en los PIJ (Strathdee y cols., 1997), y algunos autores señalan que la principal barrera para reducir los riesgos para la transmisión deI VIH/SIDA y otros riesgos para la salud entre estos pacientes consiste en el fracaso en implementar programas eficaces compatibles con los programas de tratamiento convencionales (Des Jarlais y Friedman, 1999). La primera conclusión de nuestro estudio, a la vista de las frecuencias de las conductas de riesgo para la salud observadas en el pretest es, en consonancia con otros autores (Brown y McLellan, 1996), que los programas de tratamiento para las drogodependencias en nuestro entorno deberían proporcionar servicios de educación y prevención de la transmisión del VIH y otros problemas de salud a los clientes que realizan prácticas de riesgo asociadas al uso de drogas.
Los programas para la reducción de las prácticas de riesgo asociadas a la inyección de drogas deben ir orientados a aumentar los conocimientos de los individuos sobre los riesgos para la salud asociados al consumo de drogas, su motivación para reducir sus comportamientos de riesgo y mantener los cambio ya alcanzados y su capacidad para alcanzar los cambios necesarios en su comportamiento (Insúa, 1999). Las variables mediadoras objeto de intervención en los TCMR son los conocimientos sobre los riesgos asociados al consumo inyectado de drogas, los conocimientos sobre la transmisión del VIH/SIDA y las medidas de protección frente a ésta, la motivación hacia la reducción de riesgos y la autoeficacia para afrontar diversas situaciones determinantes de las conductas de riesgo en el consumo inyectado de drogas.

Nuestros resultados confirman la existencia de un nivel de conocimientos moderado entre los UDIs acerca de los riesgos asociados a la inyección de drogas. Antes de los TCMR, tanto sus usuarios como los sujetos de comparación respondían acertadamente a un promedio de 17 ítems de los 29 (alrededor de un 59\% de las cuestiones). Los resultados de la comparación entre grupos a lo largo del estudio en estos conocimientos demuestran claramente que los usuarios de los TCMR incrementan sus conocimientos sobre la inyección de riesgo significativamente más que el grupo de comparación a lo largo del estudio en comparación con el pretest, alcanzando un nivel superior de conocimientos en el postest y seguimiento que los que no participan en los Talleres (aumentan un promedio de 5 puntos). No obstante, aún persisten tras los Talleres algunas creencias erróneas sobre estos riesgos que parecen ser resistentes al cambio.

Hemos observado también que el conocimiento sobre las formas de transmisión del VIH/SIDA y las conductas de protección frente a ésta, es moderado (los sujetos encuestados responden acertadamente a 20 de las 30 cuestiones en el pretest; alrededor del $66,7 \%)$. Estos resultados avalan que la mayoría de los sujetos drogodependientes en tratamiento que participaron en la evaluación de los TCMR mantienen algunas informaciones incorrectas sobre el VIH/SIDA. Nuestros resultados indican claramente que los usuarios de los TCMR aumentan significativamente sus conocimientos sobre la transmisión del VIH/SIDA durante los Talleres en comparación con la línea base, que lo hacen en una medida significativamente mayor que los sujetos que no participaron en dichos Talleres y que esos aumentos en los conocimientos tienden a mantenerse en el seguimiento. Aunque no se pueda asegurar que ese aumento en los conocimientos se traduzca en menores conductas de riesgo para la transmisión del VIH/SIDA, es de esperar que ese aumento tenga al menos un efecto indirecto positivo hacia la reducción de riesgos, en la línea de lo afirmado por otros autores (DiClemente, Forrest y Mickler, 
1989; Sankary, 1989) y los modelos clásicos que explican el cambio de conducta. No obstante, también nuestro estudio demuestra que algunos UDIs mantienen algunas creencias y mitos erróneos (especialmente sobre la transmisión del VIH/SIDA) resistentes al cambio, como lo demuestra el hecho de que las puntuaciones medias en los conocimientos sobre la transmisión de VIH/SIDA tras los TCMR correspondan a una tasa de aciertos promedio del $74 \%(22,2$ de 30 cuestiones).

Los resultados relativos a la motivación acerca de la reducción de los riesgos asociados al consumo inyectado de drogas muestran que, en general, los pacientes drogodependientes en tratamiento en PMM y los que acuden a PIJs mantienen altos niveles de motivación hacia la reducción de estos riesgos en el pretest. Los TCMR van asociados a un mantenimiento de esta motivación, mientras que los sujetos que no participan en estos Talleres experimentan un descenso progresivo y sustancial de esta motivación a lo largo de un periodo inferior a dos meses. Las intervenciones educativas y preventivas desarrolladas durante los TCMR están diseñadas para favorecer la implicación personal en la reducción de los riesgos asociados a la inyección de drogas y también en el mantenimiento de los progresos alcanzados, lo cual es esencial para evitar o retrasar el retorno a los antiguos patrones de conducta arriesgada (Prochaska y DiClemente, 1983, 1992).

Según la teoría del Aprendizaje Social (Bandura, 1982, Llorente, 1997) la autoeficacia de afrontamiento es una variable mediadora entre los conocimientos, las habilidades objetivas y la acción, condicionando la conducta y el esfuerzo que realiza un individuo para superar una situación determinante de una conducta arriesgada, y siendo por tanto una variable mediadora que condiciona los resultados. Nuestros resultados demuestran que los usuarios de los TCMR aumentan sustancialmente su autoeficacia para afrontar exitosamente las situaciones que determinan su conducta de riesgo en la inyección de drogas, en comparación con sujetos que no participaron en estos Talleres. La persuasión verbal, el aprendizaje y entrenamiento en nuevas habilidades de afrontamiento, la propia experiencia de afrontamiento de las situaciones de riesgo para el propio individuo y el aprendizaje vicario que se producen durante los TCMR favorecen el aumento de esta autoeficacia de afrontamiento de las situaciones de riesgo, preparando al sujeto para la puesta en práctica de las medidas de protección aprendidas.

Así pues, los resultados observados entre los usuarios de los TCMR en comparación con los no-usuarios demuestran un efecto positivo de los TCMR para aumentar o mantener el nivel de las variables mediadoras que determinan la conductas de riesgo en la inyección de drogas. Además, nuestros resultados aportan evidencia consistente que demuestra que estos Talleres reducen muy sustancial y significativamente la frecuencia de las conductas de riesgo asociadas a la inyección de drogas (reutilizar la jeringuilla ya usada: propia y/o ajena, compartir material de limpieza y proporcionar la jeringuilla a otro UDI) hasta casi hacer desaparecer algunas de ellas; que aumentan sustancial y significativamente la frecuencia de las conductas de protección frente a los riesgos en la inyección (usar siempre jeringuilla nueva) y que su efecto positivo se mantiene casi siempre en el seguimiento en comparación con los no-usuarios de estos Talleres. Creemos que el impacto de estos TCMR (porcentaje de reducción observado en las conductas de riesgo y el porcentaje de aumento en las conductas de protección) resulta especialmente reseñable, teniendo en cuenta que la intervención se aplica en pacientes en programas de tratamiento, cuyas conductas de riesgo ya habían disminuido notablemente en relación con las fases de consumo activo previas al tratamiento. Estas intervenciones breves (Talleres) pueden resultar especialmente útiles para modificar conductas de riesgo resistentes al cambio en los programas de tratamiento convencionales, siendo perfectamente compatibles con un amplio espectro de programas de tratamiento y aportando su eficacia contrastada y la reconocida eficiencia de las intervenciones grupales en el tratamiento de los trastornos adictivos.

Con respecto a la satisfacción de los usuarios con los Talleres realizados, ésta está entre notable y máxima, tanto en el postest como en el seguimiento, tanto en lo relativo a la adecuación de los mismos a las características del grupo, como a las características específicas del taller y la influencia que tiene en el cambio de conducta y a los recursos humanos utilizados en su implementación.

En resumen, podemos decir que los resultados hallados proporcionan una evidencia consistente de apoyo a la eficacia de los TCMR para el logro de sus objetivos y una elevada satisfacción de los usuarios de drogas que participan en ellos, demostrando la importancia que tiene la puesta en marcha de este tipo de Programas de reducción de riesgos en los Servicios que trabajan con usuarios de drogas.

Asimismo, demuestra que la red creada a través del Programa de Formación de Formadores ha logrado su objetivo de poner marcha programas eficaces de reducción de los riesgos asociados al consumo con los usuarios de drogas, aportando evidencia sobre la eficiencia del trabajo grupal y en redes con profesionales sanitarios.

Agradecimientos: Este trabajo ha sido realizado a través de un convenio con el Plan Nacional sobre Drogas el Plan Nacional sobre el SIDA y la Universidad del País Vasco (UPV/EHU) y se ha podido llevar a cabo gracias a todos los monitores de los Talleres, así como a los coordinadores de las distintas CC.AA. 
Ajzen, I. y Fishbein, M. (1977). Attitude and Behavior relations: A theoretical analysis and review of empirical research. Psychological Bulletin, 84: 888-918

Ajzen, I. y Fishbein, M. (1980). Understanding Attitudes and predicting Social Behavior. USA: Prentice-Hall, Inc.

Ajzen, I. y Madden, T.J. (1986). "Prediction of goal-directed behaviour: The role of intention, perceived control, and prior behaviour". Journal of Experimental Social Psychology, 26: 305-328

Bandura, A.(1982). Teoría del Aprendizaje Social. Madrid: Espasa-Calpe.

Becker, M.H. (1974). "The health beliefs model and personal health behaviour", Health Education Monographs, 2: 324-473

Catania, J.A.; Kegeles, S. y Coates, T. (1990). “Towars an understanding of risk behaviour: An Aids risk reduction model (ARRM)", Health Education Quarterly, 17, 53-72.

DiClemente, R.; Forrest, K.; Mickler, S.; (1989). Differential effects of AIDS knowledge and perceived susceptibility on the reduction of high-risk behavior among college adolescents. Fifth International AIDS Conference: Abstracts. International AIDS Association, pag. 742.

Des Jarlais, D.C.; Friedman, S.; Perlis, T. et al., (1999). “Behavior and HIV infection among new drug injectors in the era of AIDS in New York City", Journal of Acquired Immune Deficiency Syndrome Human Retrovirology, 20: $67-72$.

Donoghoe, M.; Stimson, G.; Dolan, K. (1989). "Sexual behaviour of injecting drug users and associated risks of HIV infection for non injecting sexual partners", AIDS Care, 1: $51-58$.

Ehrhardt, A.; Exner, T.; Miller, S. y Stein, Z. (1992). Sexual risk behaviour and behaviour change in heterosexual women and men. New York: Center for Clinical and Behavioural Studies at the New York State Psychiatric Institute.

Gibbons, F.; McGovern, P. y Lando, H. (1991). "Relapse and risk perception among members of smoking cessation clinic", Health Psychologist, 10: 42-45

Gibney, L.; DiClemente, R. y Vermund, S. (1999). Preventing HIV in Developing Countries. New York: Plenum Publishers

Green, L.W. y Kreuter, (1991). Health Promotion Planning: An educational and environmental approach. Mountain View, CA: Mayfield.

Insúa, P. (1999). Manual de Educación Sanitaria: Recursos para diseñar Talleres de prevención con usuarios de drogas. Madrid: Plan Nacional sobre Drogas.

Insúa, P. y Moncada, S. (2000a). "Opinión expresada sobre un programa de formación de formadores para profesionales que trabajan con UDIs", Adicciones, 12, 4: 479486

Insúa, P. y Moncada, S. (2000b). "Cómo diseñar un programa de prevención de la transmisión sexual del VIH para usuarios de drogas?". Trastornos Adictivos, 2, 3: 201210

Insúa, P. y Moncada, S. (2001). "¿Está cambiando el discurso sobre el uso de drogas en las Instituciones?: Un Programa de Formación de Formadores". Revista Española de Drogodependencias, Vol. 26, 1: 6-28

Insúa, P. Lledó, M y Grijalvo, J. (2001). "Nuevos conceptos, nuevo discurso: Talleres de consumo de menos riesgo." Trastornos Adictivos, 3, 2: 111-120.

Insúa, P. (2002). Evaluación de los Talleres de Sexo Más Seguro y Consumo de Menos Riesgo para Usuarios de Drogas. Características Psicométricas de los Instrumentos de Medida. Documento no publicado. Madrid: Delegación del Gobierno para el Plan Nacional sobre Drogas.

Kline, A.; Strickler, J. (1993). "Perceptions of risk for AIDS among women in drug treatment." Health Psychology, 12, 4: 313-323.

Llorente, J.M. (1997). Validación del DTCQ-H en la población española. Tesis doctoral no publicada. Madrid: Universidad Nacional de Educación a Distancia.

Mantell, J.; DiVittis, A. y Auerbach, M. (1997). Evaluating HIV Prevention Interventions. New York: Plenum Press.

Marlatt, A. (1998). Harm Reduction. Pragmatic Strategies for Managing High-Risk Behaviors New York: The Guilford Press

Murphy, D. (1989). "Heterosexual contacts of intravenous drug abusers: implications for the next spread of the AIDS epidemic". AIDS and Substance Abuse, 89-97.

Prochaska, J.O. y DiClemente, C.C. (1983). "Stages and processes of self-change of smoking: Toward and integrative model of change." Journal of Consulting and Clinical Psychology, 51: 390-395.

Prochaska, J.O. y DiClemente, C.C. (1992). "Stages of change in the modification of problem behaviors". En: M. Hersen, R.M. Eisler y P.M. Miller (editores): Progress in behaviour modification. Sycamore, Illinois: Sycamore Press.

Sankary, T.; (1989). "The impact of teenager's knowledge and concern of AIDS on sexual behavior and prevention strategies." Fifth International AIDS Conference: Abstracts. International AIDS Association, pag. 727.

Schifter, D.E. y Ajzen, I. (1985). "Intention, perceived control and weight loss: An application of the theory of planned behaviour". Journal of Personality and Social Psychology, 49: 843-851.

Stimson, G.V.; Power, R.; (1992). "Assessing AIDS prevention for injecting drug users: some methodological considerations." British Journal of Addiction, 87, 3: 455-465.

Stimson, G.V. (1995). "AIDS and Injecting Drug Use in the United Kingdom, 1987-1993: The Policy Response and the Prevention of the Epidemic." Social Science Medicine, 41, 5: 699-716. 
Strathdee, S.A.; Patrick, D.M.; Currie, S.L.; Cornelisse, P.G.A.; Rekart, M.L.; Montaner, J.S.G.; Schechter, M.T.; Oshaughnessy, M.V. (1997). "Needle exchange is not enough: Lessons from the Vancouver injecting drug use study". AIDS. 11, 8: 59-65.

Watters, J.; Estilo, M.; Kral, A.; et al. (1994). HIV infection among female injection-drug users recruited in com- munity settings. Sexually Transmitted Diseases, 21(6): 321-328.

Watters, J.; Estilo, M.; Clark, G.L.; Lorvick, J. (1994). Syringe and needle exchange as HIV / AIDS prevention for injection drug users. JAMA, 271(2): 115-120. 